\title{
Aportes introductorios a la reflexión sobre gobernanza y reforma del sector seguridad
}

\author{
https://doi.org/10.21830/9789585350601.01
}

\author{
Mateo Morales Callejas ${ }^{l}$ \\ Ximena Andrea Cujabante Villami \\ Universidad Militar Nueva Granada \\ Manuel Alexánder Betancur Montoya ${ }^{3}$ \\ Escuela Militar de Cadetes "General José María Córdova”
}

El contexto nacional de transición obliga a pensar en la necesidad de hacer importantes modificaciones en los aparatos de seguridad y defensa del Estado colombiano, los cuales han debido dedicar gran parte de sus esfuerzos a librar la guerra a raíz del desarrollo del conflicto armado interno. La fase de posacuerdo con la guerrilla de las Farc conduce, casi inevitablemente, a que desde la práctica y la discusión conceptual se piense cómo deben reformarse tanto la Policía Nacional como las Fuerzas Militares del país. De aquí que el concepto de gobernanza y reforma del sector seguridad cobre particular relevancia.

Las reflexiones y prácticas en torno a la necesidad de reformar el sector seguridad y defensa de los países son herederas de una serie de cambios en el escenario internacional. De una parte, el fin de la Guerra Fría dio lugar a la transformación y la ampliación del concepto de la seguridad, así como a

1 Politólogo y magíster en Ciencia Política de la Universidad de los Andes. Orcid: https://orcid. org/0000-0001-5272-1959.

2 Politóloga de la Universidad Javeriana, especialista en Negociación y Relaciones Internacionales de la Universidad de los Andes, magíster en Asuntos Internacionales y doctora en Estudios Políticos de la Universidad Externado de Colombia. Docente de tiempo completo de la Facultad de Relaciones Internacionales, Estrategia y Seguridad de la Universidad Militar Nueva Granada. Orcid: https://orcid. org/0000-0002-5473-163X. Contacto: ximena.cujabante@unimilitar.edu.co

3 Magister en Estudios Políticos e Internacionales, Universidad del Rosario. Profesional en Relaciones Internacionales y Estudios Políticos, Universidad Militar Nueva Granada. Profesional en Ciencias Militares, Escuela Militar de Cadetes "General José María Córdova". Orcid: https://orcid.org/0000-0001$6511-9000$ 
cambios significativos en el desarrollo práctico de las políticas de seguridad y defensa. En especial, la visión convencional de la seguridad cimentada en el Estado como unidad de análisis y de los asuntos estratégico-militares como fuente principal de amenaza fue reorientada hacia el individuo y las comunidades. Dicha evolución es ejemplificada por conceptos como la seguridad humana y la seguridad ciudadana, que amplían el espectro de los desafíos existentes al bienestar de las personas, precisan una transformación fundamental de las funciones de la Fuerza Pública y enfatizan una provisión de la seguridad orientada a imperativos tales como la legitimidad, la transparencia y la democratización. Pese a que la seguridad ciudadana, a diferencia de la humana, busca acotar el tipo de amenaza a fenómenos como el delito y la violencia, que restringen el ejercicio de las libertades y los derechos democráticos de las personas, en ambos casos se considera vital el compromiso cívico y la rendición de cuentas de la Fuerza Pública frente a las ramas ejecutiva, legislativa y judicial, así como ante las autoridades locales y los ciudadanos, en un modelo ascendente (o bottom-up) de diseño, ejecución y control de las políticas de seguridad y defensa $a^{4}$.

De otra parte, en concordancia con los intentos académicos y políticos de los años noventa de promover una aproximación ampliada a la seguridad centrada en las personas, en aquellos contextos de transición política del autoritarismo a la democracia, y de la guerra a la paz, se comenzó a insistir en la necesidad de incorporar la reforma de las instituciones de la seguridad y la defensa dentro de los procesos de construcción de paz. La comunidad internacional comenzó a prestar atención a la necesidad de reformar, transformar y generar gobernanza del "sector seguridad" en aquellas sociedades que lograban finalizar sus conflictos, como parte integral de los procesos de construcción de paz (Schroeder, Chappuis \& Kocak, 2014). En 2005, la Organización de Naciones Unidas (Onu) reconoció que la gobernanza y la reforma del sector seguridad eran pasos esenciales para los procesos de estabilización en sociedades en posconflicto (Schnabel \& Ehrhart 2005; Sedra, 2006, 2017).

4 Para una discusión más detallada sobre los debates actuales en torno a la seguridad en la región latinoamericana, véase Programa de las Naciones Unidas para el Desarrollo (PNUd, 2013). 
Desde la óptica de organizaciones internacionales como la Onu, la Organización para la Cooperación y el Desarrollo Económicos (Ocde, 2005, 2007), la Organización para la Seguridad y la Cooperación en Europa (Osce, 2016), think thanks como el Centro de Ginebra para la Gobernanza del Sector de la Seguridad (Dcaf, 2015), y siguiendo las argumentaciones de diferentes autores que han abordado el tema de la gobernanza y la reforma del sector seguridad (Grss) (Schnabel \& Ehrhart, 2005; Sedra 2006, 2017; Schroeder et al., 2014), la reforma del sector seguridad se vuelve fundamental en las sociedades en posconflicto o estabilización, sustancialmente porque al término de la guerra las instituciones de seguridad y defensa están alteradas por el mismo desarrollo del enfrentamiento: presentan elevados niveles de subdesarrollo, solapamiento entre policías y militares, malas y deterioradas relaciones con las comunidades, abusos sobre la población civil, entre otros problemas.

En este sentido, el concepto de reforma al sector seguridad emergió como complemento a las aproximaciones tradicionales a la cooperación internacional prestada por distintos países desarrollados y organismos multilaterales. Los objetivos principales de la Grss giran en torno a la creación de esquemas de buen gobierno en el sector seguridad e incluyen: la promoción de la efectividad y la eficiencia en el suministro de la seguridad y la justicia; la transformación de las funciones de las Fuerzas Armadas acorde con las necesidades de la ciudadanía; la construcción de una fluida relación cívico-militares/policiales; el fortalecimiento del control civil sobre el sector y el mejoramiento de las capacidades de los civiles de incidir en la toma de decisiones en seguridad y defensa, todo dentro del marco de la democracia, el Estado de derecho, la transparencia, la rendición de cuentas y el respeto por los Derechos Humanos5.

Pese a la relevancia temática y política del concepto, en Colombia son escasas las reflexiones que se han generado en torno a este debate. Situación que no deja de ser paradójica teniendo en cuenta que durante más de cincuenta años el país ha estado enfrascado en una lógica en la que los temas de seguridad y defensa permean el día a día. Tal vez una posible respuesta radique precisamente en la sensibilidad de este asunto, lo cual lo ha relegado en las manos de

5 Para profundizar en este debate, véase Schnabel y Ehrhart (2005), Labarre y Tzankova (2011); Gsdr y GFN-SsR (2013) y Ball (2014). 
unos pocos expertos que se encargan de su manejo, conceptualización y ejecución. Este déficit en la discusión llega a ser más preocupante si se mira desde la óptica de los medios de comunicación, ya que además de que no se comparte información (oficial u otra) con ellos, tampoco exhiben mayor interés por estos temas. Una posible explicación de esta situación puede ser, precisamente, el hecho de que las discusiones sobre seguridad y defensa se han desarrollado en un contexto de guerra permanente, lo cual ha llevado a que solo las Fuerzas Armadas y unos pocos civiles sean los encargados de manejarlo, de manera que la sociedad civil se ha alejado del debate. Sin embargo, y más allá del posible monopolio del tema que tienen los sectores militares y policiales, en Colombia ha habido un desinterés palpable por parte de la sociedad en general y de algunos sectores políticos en abordar, de forma seria, los ámbitos de la seguridad y la defensa (Morales \& Tickner, 2019).

En un contexto de transición de la guerra a la paz este desinterés no deja de ser sorpresivo. Si no existe un debate público informado es poco probable que se pueda consolidar una hoja de ruta clara de cómo, hacia dónde y con qué objetivos se deben transformar las instituciones de seguridad del Estado, que si bien están muy bien equipadas y entrenadas para el combate contra los actores armados, carecen de un norte claro en cuanto al desarrollo de otras funciones. En ese sentido, varios de los capítulos de este libro discuten elementos o componentes de diferente orden que podrían ser tenidos en cuenta a modo de recomendación de cara a una posible reforma al sector seguridad y defensa en Colombia.

Con miras a enriquecer la literatura producida hasta el momento, este libro recoge el trabajo de un grupo de profesores e investigadores tanto de la Universidad Militar Nueva Granada como de la Universidad del Rosario (en el marco de un convenio de investigación firmado entre las dos universidades) sobre las distintas facetas de la reforma y gobernanza del sector seguridad y defensa. Vale la pena agradecer la participación de la profesora Arlene B. Tickner como coordinadora editorial, quien con su asesoría académica direccionó la discusión temática para que este libro llegara a buen término. Asimismo, se contó con la participación de docentes investigadores de la Facultad de Relaciones Internacionales, Seguridad y Estrategia de la 
Universidad Militar Nueva Granada y de asistentes de investigación, quienes en un esfuerzo mancomunado lograron a través de este libro ofrecer insumos variados y complementarios a partir de enfoques interdisciplinarios.

Además de ahondar en algunos aspectos subexplorados de la literatura especializada, el principal aporte del libro radica en que visibiliza un tema de gran sensibilidad para nuestro contexto, de una manera informada, ofreciéndole al lector insumos para su estudio futuro, más allá de los análisis coyunturales y descriptivos. En ese orden de ideas, el presente volumen consta de cinco capítulos, que, de formas diferenciadas, abordan el tema de la reforma y la gobernanza del sector seguridad y defensa. Estas reflexiones no se centran, de manera exclusiva, en el caso colombiano, sin embargo, sí generan aportes significativos para pensar cómo debería ser el proceso de reforma y gobernanza del sector en nuestro país, teniendo en cuenta que este todavía está a ciernes de consolidarse.

Como se verá en los capítulos subsiguientes, la conceptualización de la reforma y la gobernanza del sector seguridad sigue siendo debatida (como lo ha sido y sigue siendo el concepto de seguridad). Pese a ello, el libro adopta una definición que incluye los siguientes elementos:

1. La administración, supervisión y control de la política y provisión de servicios de seguridad y justicia por parte de los actores del sistema/ sector seguridad, que (i) aplica los principios de la buena gobernanza (i.e., administración efectiva, equitativa, sensible y transparente de los asuntos y recursos públicos y rendición de cuentas a autoridades civiles democráticas); (ii) es compatible con el imperio de la ley; (iii) asegura el control civil democrático de los asuntos de seguridad a través de la participación informada de agentes de las tres ramas del poder estatal y de la sociedad civil en la formulación, implementación y control de políticas del sistema/sector seguridad, con una orientación clara hacia las normas y reglas formuladas a nivel regional y global.

2. Igualmente, la conceptualización incluye los conjuntos de planes, programas, procesos, medidas y/o actividades políticas y técnicas 
nacionales, regionales y globales que, en contextos de transición política y mediante un reconocimiento comprehensivo de las diversas amenazas y objetos encapsulados por visiones amplias de seguridad propenden a (i) incrementar la habilidad de los sistemas/sectores de seguridad para realizar, administrar, supervisar y controlar la provisión de servicios de seguridad y justicia de una manera que responda con efectividad frente al rango de necesidades de seguridad de individuos y comunidades y (ii) asegurar que dicha provisión de servicios sea consistente y compatible con el respeto por los Derechos Humanos, el desarrollo humano y la igualdad de género, como también con mecanismos adicionales de justicia transicional, control de armas, iniciativas anticorrupción o combate al crimen organizado, de tal manera que se procura una mayor gobernanza del sector seguridad, relaciones cívico-militares constructivas y/o la consolidación de la paz después del conflicto a largo plazo.

En ese orden de ideas, el libro inicia con un capítulo que indaga conceptualmente en relación con los conceptos de reforma al sector seguridad (Rss) y gobernanza del sector seguridad (Gss). La revisión sistemática de literatura desarrollada por el autor le permite identificar el origen histórico de los conceptos y las formas como diversos actores de la comunidad internacional han tendido a utilizarlos, teniendo en cuenta que estos han surgido atados a los procesos de ayuda internacional. Igualmente, muestra de manera clara cuáles son los debates académicos actuales frente a estos conceptos y la forma como se han manifestado en diferentes casos de estudio. Finalmente, y de gran relevancia, el autor expone cuáles podrían ser considerados como indicadores de éxito de los procesos de reforma y gobernanza del sector seguridad.

El segundo capítulo aborda los principales retos y tendencias de la reforma de la seguridad y la defensa que debe enfrentar Colombia. Se pone de presente cómo ciertos temas, tales como la ausencia de una normativa clara de seguridad y defensa; la confusión de roles entre policías y militares a raíz del conflicto armado; la existencia de un distanciamiento entre la sociedad civil y los sectores de la seguridad y la defensa, y la falta de atención a las necesidades territoriales 
en materia de seguridad constituyen los principales retos que se deben abordar ante un eventual proceso de reforma al sector en Colombia. Igualmente, se propone un elemento adicional que se debería considerar tanto en la reflexión conceptual y académica de la Grss, como entre los retos del país: la internacionalización. El capítulo evidencia cómo la internacionalización es un componente visible en la práctica colombiana de reformar su sector de seguridad y que, en gran medida, cuestiona ciertos presupuestos teóricos del concepto.

La territorialidad, la construcción de paz y la reforma al sector seguridad son los temas centrales del tercer capítulo. A partir de una reflexión conceptual en torno al enfoque de paz territorial y su importancia de cara a la gestión de la seguridad y la convivencia en el posacuerdo, se exponen las experiencias de un proyecto de la Policía Nacional circunscrito en el Modelo de Construcción de Paz de la institución. El capítulo pone en evidencia los principales retos al territorializar los temas de seguridad y convivencia ciudadana, y sugiere que es necesario prestar atención a los avances que la Policía ha realizado en esta materia, de modo tal que se puedan replicar y tenerse en cuenta en procesos más amplios de reforma del sector seguridad y defensa en Colombia.

Pese a la relevancia que tiene la seguridad y la defensa en nuestro país, existe un enorme vacío que ha sido identificado de distintas maneras, tanto en el campo político como en el académico: no existe una guía clara en materia de seguridad y defensa que trascienda las políticas de gobierno y se constituya como una hoja de ruta de Estado. En ese sentido, el cuarto capítulo propone un estudio comparado desde el derecho que permite reflexionar en torno a los elementos normativos que debería poseer una ley de seguridad y defensa en Colombia de cara a un eventual proceso de reforma del sector. Así, se abordan las leyes de seguridad y defensa de Chile, Argentina y Brasil para extraer de allí los elementos básicos que debería incluir un libro blanco de defensa colombiano, que es necesario para que el país afronte los importantes retos de seguridad que se asoman en el escenario actual.

El último capítulo del libro hace un estudio comparado de las reformas al sector seguridad y las relaciones cívico-militares en cuatro países de América Latina. A partir del meticuloso estudio de las experiencias de Perú, Chile, El Salvador y Guatemala, se esboza la variedad de aspectos que caracterizan los 
procesos de reforma del sector seguridad en contextos de transición política. En cada uno de los casos, se aborda el contexto histórico que explica la injerencia política de las Fuerzas Armadas en los distintos países, para luego comparar y contrastar los procesos de reforma del sector seguridad en cada uno de ellos a través de seis variables de análisis.

Se espera que la presente publicación, producto de un trabajo conjunto e interdisciplinar, contribuya a socializar y aclarar en el país el concepto de gobernanza y reforma del sector seguridad (GRSs), teniendo en cuenta su relevancia en procesos de transición política. El escenario colombiano actual plantea una serie de retos y oportunidades en materia de seguridad y defensa que se deben abordar de forma oportuna, en función de no seguir reproduciendo algunas lógicas poco eficientes y contraproducentes en el manejo de la seguridad y la defensa del país. Si bien es claro que se requieren procesos de mejora (técnica y profesional) y transformaciones doctrinarias de cara a los "nuevos" retos de seguridad del contexto actual, también es clave no perder de vista que este tema no se debe dejar a la deriva ni bajo el manejo exclusivo de las instituciones de seguridad, teniendo en cuenta que muchas de las problemáticas territoriales que enfrenta la Colombia del posacuerdo tienen un importante, por no decir primario, componente de orden socioeconómico.

Es indispensable, además, comenzar a hacer público el debate de la seguridad y la defensa y de sus procesos de reforma, ya que la seguridad y la defensa son bienes públicos frente a los cuales es importante y deseable generar rendición de cuentas y control civil. Varias de las experiencias de reforma a las Fuerzas Militares y a la Policía Nacional que existen hoy en día siguen siendo laboratorios cerrados, frente a los cuales perduran innumerables dudas, como por ejemplo cómo están siendo financiados; qué entidades están marcando las pautas de las transformaciones; cuál es el papel que tiene la cooperación internacional en todo el proceso, entre otros interrogantes. Finalmente, hacer público el debate de la Grss permitirá rescatar todos los esfuerzos que están haciendo las mismas instituciones de seguridad y, sobre todo, aquellas unidades a la vanguardia de las problemáticas actuales, de modo tal que se puedan articular de forma eficiente y orientada a objetivos concretos. 


\section{Referencias}

Ball, N. (2014). Putting governance at the heart of security sector reform. Clingendael Netherlands Institute of International Relations.

Geneva Centre for the Democratic Control of Armed Forces [Dcaf]. (2015). Security Sector Reform. Applying the principles of good governance to the security sector. Geneva Centre for the Democratic Control of Armed Forces.

Gsdr, \& Gfn-Ssr. (2013). Topic guide on security sector reform. Governance and Social Development Resource Centre; Global Facilitation Network for Security Sector Reform.

Labarre, F., \& Tzankova, M. (eds.). (2011). Theoretical and technical perspectives on security sector governance. Ios Press.

Morales, M., \& Tickner, A. (2019). ¿Qué sector seguridad necesita Colombia? Dilemas de la gobernanza de la seguridad y la defensa en el posconflicto. Fescol.

Organización para la Cooperación y el Desarrollo Económicos [Ocde]. (2005). Security System Reform and Governance. A Dac Reference Document. Oecd Publishing.

Organización para la Cooperación y el Desarrollo Económicos [Ocde]. (2007). OECD DAC Handbook on Security System Reform. Supporting Security and Justice. Oecd Publishing.

Organización para la Seguridad y la Cooperación en Europa [Osce]. (2016). Security Sector Governance and Reform. Guidelines for Osce Staff. Osce.

Programa de las Naciones Unidas para el Desarrollo [Pnud]. (2013). Informe regional de desarrollo humano 2013-2014. Seguridad ciudadana con rostro humano: diagnóstico y propuestas para América Latina. Pnud.

Schnabel, A., \& Ehrhart, H. (2005). Security sector reform and post-conflict peacebuilding. United Nations University Press.

Schroeder, U., Chappuis, F., \& Kocak, D. (2014). Security sector reform and the emergence of hybrid security governance. International Peacekeeping, 21, 214-230.

Sedra, M. (2006). European approaches to security sector reform: Examining trends through the lens of Afghanistan. European Security, 15(3), 323-338.

Sedra, M. (2017). Security sector reform in Conflict-Affected Countries: The evolution of a model. Routledge. 